EPJ Web of Conferences 49, 18018 (2013)

DOI: $10.1051 /$ epjconf/20134918018

(C) Owned by the authors, published by EDP Sciences, 2013

\title{
Search for a Higgs boson produced in the vector boson fusion process in the diphoton channel with the ATLAS experiment
}

\author{
Elisabeth Petit ${ }^{1, a}$ on behalf of the ATLAS Collaboration \\ ${ }^{1}$ LAPP, CNRS/IN2P3 and University of Savoie, Annecy-le-Vieux, France
}

\begin{abstract}
Details of the production of the new particle discovered by the ATLAS experiment at the LHC in the mass region of $m_{H}=126 \mathrm{GeV}$ in the search for the Standard Model Higgs boson at $\sqrt{s}=7 \mathrm{TeV}$ and 8 $\mathrm{TeV}$ are presented. The addition of a category enriched in vector boson fusion events in the $H \rightarrow \gamma \gamma$ search allows to increase the sensitivity of the search of a Standard Model Higgs boson. It also allows to separate better between gluon fusion and vector boson production modes, thus giving a first insight to the couplings of this newly discovered boson.
\end{abstract}

\section{Introduction}

The ATLAS [1] and CMS [2] collaborations have independently reported observations of a new boson compatible with the SM Higgs boson, using the 2011 and 2012 datasets collected until the end of June 2012 [3, 4]. More detailed measurements of this particle is necessary in order to check the compatibility with the Standard Model predictions, in particular the study of the several production modes. These production modes are, by decreasing order of cross-section production: gluon-gluon fusion (noted as $g g \mathrm{~F})$, vector boson fusion $(\mathrm{VBF})$, associated production with a vector boson $(V H)$ and associated production with a $t \bar{t}$ pair $(t \bar{t} H)$. The expected cross-sections for those four processes, for a center-of-mass energy of $8 \mathrm{TeV}$ and a Higgs mass of $125 \mathrm{GeV}$ are $44.5 \mathrm{fb}, 3.6 \mathrm{fb}, 2.5 \mathrm{fb}$ and 0.9 $\mathrm{fb}$ respectively, when multiplied by the $H \rightarrow \gamma \gamma$ branching ratio $\left(2.28 \times 10^{-3}\right)$.

The addition of a category enriched in VBF events allows to improve the sensitivity of the Higgs boson search as well as giving tools to give a first estimate of the couplings of this new boson to fermions and bosons.

\section{Event selection}

The dataset used in this study is based on $4.8 \mathrm{fb}^{-1}$ of $\sqrt{s}$ $=7 \mathrm{TeV}$ data recorded in 2011 and $5.9 \mathrm{fb}^{-1} \sqrt{s}=8 \mathrm{TeV}$ data recorded in the first months of 2012 [5].

At least two photons satisfying tight identification criteria based on the shapes of the EM showers are required. The transverse energies for the leading and subleading photons are required to be larger than $40 \mathrm{GeV}$ and $30 \mathrm{GeV}$, respectively, and both need to be within the fiducial calorimeter region of pseudorapidity $|\eta|<2.37$ (excluding the transition region between the barrel and the end-cap calorimeters, $1.37<|\eta|<1.52$ ).

a e-mail: Elisabeth.Petit@ cern.ch

\begin{tabular}{c||c|c|c|c|c||c}
\hline$\sqrt{s}$ & ggF & VBF & VH & $t \bar{t} H$ & total & data \\
\hline $7 \mathrm{TeV}$ & 70.9 & 5.8 & 3.7 & 0.3 & 80.7 & 23788 \\
$8 \mathrm{TeV}$ & 100.3 & 8.3 & 5.0 & 0.5 & 114.1 & 35271 \\
\hline
\end{tabular}

Table 1. Number of events in the data and number of expected signal events for $m_{H}=125 \mathrm{GeV}$ [5]

In addition to the identification criteria, both photons are also required to be isolated: the transverse energy sum of positive-energy topological clusters deposited in the calorimeter around each photon in a cone of $\Delta R^{1}=$ 0.4 , and is required to be less than $4 \mathrm{GeV}$. The resolution of the diphoton mass is dominated by the photon energy resolution.

The number of selected data events and expected signal events for $m_{H}=125 \mathrm{GeV}$ after the full selection is given in table 1.

\section{Categorisation}

The data is divided into 10 exclusive categories with different signal-to-background ratios and different invariant mass resolution. This increases the sensitivity of the search. The first nine categories are described in [5] and are based on the information of the $\eta$ and conversion status of the photons, as well as the diphoton transverse momentum orthogonal to the diphoton thrust axis in the transverse plane $\left(p_{\mathrm{Tt}}\right)$. The use of the categories based on $p_{\mathrm{Tt}}$ already gives a separation between $\mathrm{VBF}$ and $g g \mathrm{~F}$ events, the former having larger $p_{\mathrm{Tt}}$ values. The tenth category is based on jets and described in the following.

\footnotetext{
${ }^{1} \Delta R=\sqrt{\eta^{2}+\varphi^{2}}$, where $\varphi$ is the azimuthal angle around the beam line
} 


\subsection{Definition of the 2-jet category}

The definition of a VBF-enriched category (called "2-jet category") is based in the kinematic properties of the VBF for which two forward jets are produced. Jets are reconstructed from three-dimensional clusters of energy in the electromagnetic and hadronic calorimeters using the anti$k_{t}$ [6] algorithm with a distance parameter of $\mathrm{R}=0.4$. Jets candidates are required to have a transverse momentum greater than $25 \mathrm{GeV}$, expect for the $\sqrt{s}=8 \mathrm{TeV}$ data where the threshold is $30 \mathrm{GeV}$ for $2.5<|\eta|<4.5$.

This category collects events containing at least two jets with a pseudorapidity separation $|\Delta \eta|$ larger than 2.8 and an invariant mass greater then $400 \mathrm{GeV}$. In addition, the azimuthal angle difference $|\Delta \varphi|$ between the diphoton and the dijet systems is required to be larger than 2.6. Those variables are presented in figures 1 and 2 for VBF and $g g \mathrm{~F}$ produced signal as well as background. One can see that those variables are used to distinguish between $\mathrm{VBF}$ and $g g \mathrm{~F}$ as well as between VBF and background.

As those cuts, the fraction of VBF events in this category is of $76.7 \%$ and $68.4 \%$ for the $\sqrt{s}=7$ and $8 \mathrm{TeV}$ respectively.

\subsection{Signal properties and uncertainties}

Figure 3 shows the diphoton invariant mass $\left(m_{\gamma \gamma}\right)$ distribution in the "2-jet category" for the $\sqrt{s}=7$ and $8 \mathrm{TeV}$ data events. For the $\sqrt{s}=8 \mathrm{TeV}$ processes, the signal-tobackground ratio in a window containing $90 \%$ of the signal is 0.22 , which has to be compared to 0.03 in the inclusive case. This high signal-to-background ratio explains the increase of sensitivity due to this category. The mass resolution (FWHM) is this category is $3.7 \mathrm{GeV}$, similar to the one in the inclusive case $(3.9 \mathrm{GeV})$.

The introduction of jets to define this category added uncertainties related to them. The main one comes from the perturbative uncertainty on the gluon fusion contribution to this category, and is found to be $25 \%$. The production cross-section of a Higgs boson via gluon fusion associated with two jets is only know at the Next-to-Leading Order, and higher-order logarithmic contributions are presented in this limited region of phase space. This large uncertainty is fortunately compensated by the small contribution of $g g \mathrm{~F}$ events in the category $(22.5 \%$ and $30.4 \%$ for $\sqrt{s}=7 \mathrm{TeV}$ and $8 \mathrm{TeV}$ respectively). The uncertainty due to the modelling of the underlying event is estimated by comparing different underlying event tunes in the simulation. For the $2-$ jets category, a $30 \%$ uncertainty is assigned to the contribution from $g g \mathrm{~F}, V H$ and $t \bar{t} H$, and a $6 \%$ uncertainty is assigned to the contribution from VBF. The experimental uncertainties on the jet energy scale is evaluated by varying the scale corrections within their respective uncertainties. The uncertainty for the different classes of categories and different production processes amount to up to $19 \%$ for the "2-jet category".

\section{Results}

The statistical procedures used to test the background-only hypothesis takes the form of statistical tests of different hy- pothesized values of a strength parameter $\mu$, defined as the ratio of the signal rate (cross section) being tested to that predicted by the SM. That is, $\mu=0$ is the background-only hypothesis and $\mu=1$ is the SM hypothesis. At fixed values of the Higgs boson mass $m_{H}$, different values of $\mu$ are tested using a statistic based on the profile likelihood ratio. To quantify discovery significance, the $p$-value of the background-only hypothesis, $p_{0}$, is reported and can be seen in figure 4. Equivalently, this can be expressed using the discovery significance. By using an inclusive study, the expected (observed) significance is $1.9 \sigma(3.5 \sigma)$, whereas by using the 10 exclusive categories, the expected (observed) significance is $2.4 \sigma(4.7 \sigma)$. Adding a tenth category improves the expected significance by a few percents.

\section{Higgs boson coupling properties}

Because of a category sensitive to the VBF, it is possible to study the contributions from the different production modes, in order to assess any tension between the data and the ratios of the production cross-sections in the Standard Model. For each production mode, a signal strength is defined, which is defined by $\mu_{i}=\sigma_{i} / \sigma_{i, S M}$. Production modes have been grouped together: $\mu_{g g F}$ with $\mu_{t \bar{t} H}$ as they scale with the $t \bar{t} H$ coupling, and $\mu_{V B F}$ with $\mu_{V H}$ as they scale with the $W W H$ and $Z Z H$ couplings in the Standard Model. Constraints in the plane of $\mu_{g g F+t \bar{t} H} \times$ $B / B_{S M}$ (where $B$ is the branching ratio for $H \rightarrow \gamma \gamma$ ) and $\mu_{V B F+V H} \times B / B_{S M}$ can be seen in figure 5 , for $m_{H}=126$ $\mathrm{GeV}$. The data are compatible with the Standard Model expectation at the $1.5 \sigma$ level.

The coupling properties of the boson can be further studied by combining results from several decay channels: $H \rightarrow \gamma \gamma, H \rightarrow Z Z, H \rightarrow W W, H \rightarrow \tau \tau$, and $H \rightarrow b \bar{b}$ [7]. The leading order (LO) motivated scale factors $\kappa_{i}$ are defined in such a way that the cross sections $\sigma_{i i}$ and the partial decay widths $\Gamma_{i i}$ associated with the SM particle $i$ scale with the factor $\kappa_{i}^{2}$ when compared to the corresponding SM prediction.

For example, among the production modes, $\kappa_{g}^{2}=\frac{\sigma_{g g F}}{\sigma_{g g F}^{2}}$, $\kappa_{W H}^{2}=\frac{\sigma_{W H}}{\sigma_{W H}^{2}}$, etc, and among the decay modes, $\kappa_{\gamma}^{2}=\frac{\Gamma_{\gamma \gamma}}{\Gamma_{\gamma}^{2}}$, $\kappa_{W}^{2}=\frac{\Gamma_{W W^{(*)}}}{\Gamma_{W W^{(*)}}^{2}}$, etc.

The couplings to fermions and vector bosons are studied by assuming scale factors $\kappa_{F}$ for the couplings to all fermions and $\kappa_{V}$ for the couplings to all vector bosons:

$$
\begin{aligned}
& \kappa_{F}=\kappa_{t}=\kappa_{b}=\kappa_{\tau} \\
& \kappa_{V}=\kappa_{W}=\kappa_{Z}
\end{aligned}
$$

By assuming that the total width of the Higgs boson is given by the sum of the known SM Higgs boson decay modes, the constraints in the $\kappa_{F}$ and $\kappa_{V}$ plane are given in figure 6. The $68 \% \mathrm{CL}$ intervals when profiling over all other parameters are: $\kappa_{F} \in[-1.0,-0.7] \cup[0.7,1.3]$ and $\kappa_{V} \in[0.9,1.0] \cup[1.1,1.3]$. The compatibility of the SM hypothesis with the best fit point is $21 \%$. 


\section{References}

[1] ATLAS Collaboration, JINST 3 S08003 (2008)

[2] CMS Collaboration, JINST 3 S08004 (2008)

[3] CMS Collaboration, Phys.Lett.B716, 30-61 (2012), arXiv:1207.7235
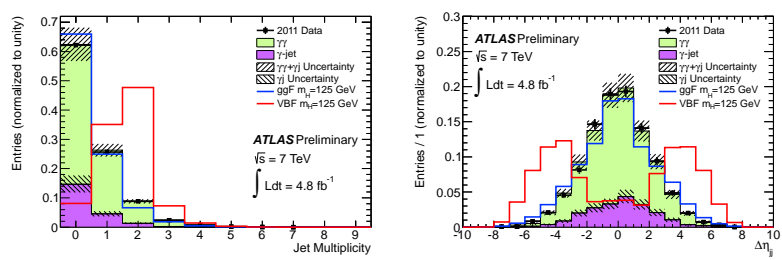

Figure 1. Jet multiplicity and $\eta$ separation of the leading and subleading jets for $\sqrt{s}=7 \mathrm{TeV}$ compared to simulation. The diphoton component is simulated with SHERPA, while the gammajet component is simulated with Alpgen, and the small jet-jet and Drell-Yan components are neglected. The distributions are normalized to unit area [5].
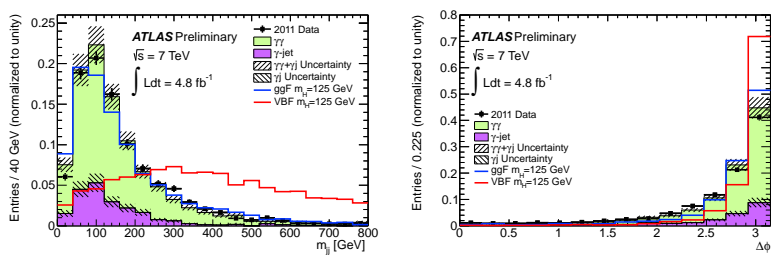

Figure 2. Dijet invariant mass and $\varphi$ separation between the diphoton and the dijet system for $\sqrt{s}=7 \mathrm{TeV}$ compared to simulation. The diphoton component is simulated with SHERPA, while the gamma-jet component is simulated with ALPGEN, and the small jet-jet and Drell-Yan components are neglected. The distributions are normalized to unit area [5].
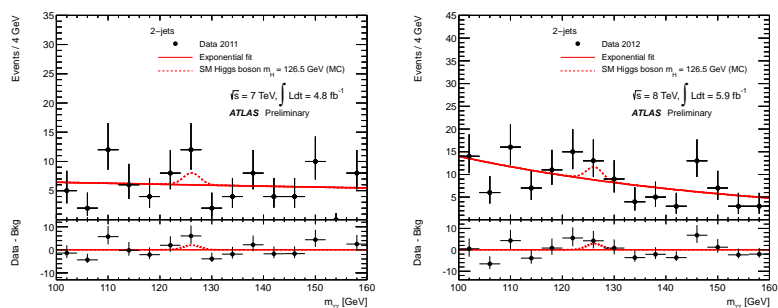

Figure 3. Background-only fit to the diphoton invariant mass spectra for the category 2-jets, for $\sqrt{s}=7 / 8 \mathrm{TeV}$. The bottom inset displays the residual of the data with respect to the background fit. The Higgs boson expectation for a mass hypothesis of $126.5 \mathrm{GeV}$ corresponding to the $\mathrm{SM}$ cross section is also shown [5].
[4] ATLAS Collaboration, Phys.Lett.B716, 1-29 (2012), arXiv: 1207.7214

[5] ATLAS Collaboration, ATLAS-CONF-2012-091 (2012), http://cds.cern.ch/record/1460410

[6] M. Cacciari, G.P. Salam, G. Soyez, JHEP 0804 (2008)

[7] ATLAS Collaboration, ATLAS-CONF-2012-127 (2012), http://cds.cern.ch/record/1476765

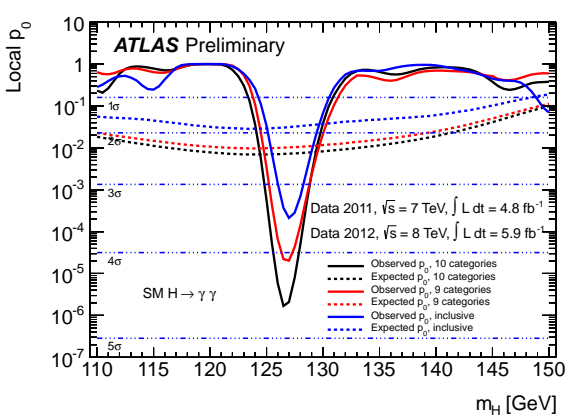

Figure 4. Expected and observed local $\mathrm{p} 0$ for the analysis using 10 categories, compared to an analysis using only 9 categories (no 2-jets category) and a fully inclusive analysis for the combined $\sqrt{s}=7 \mathrm{TeV}$ and $\sqrt{s}=8 \mathrm{TeV}$ data [5].

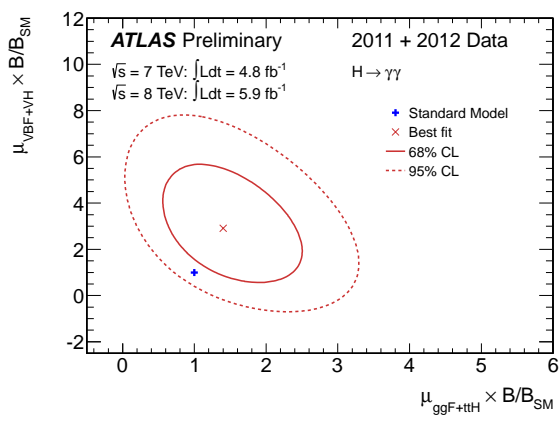

Figure 5. Measurements of the signal strength parameter $\mu$ for $\mathrm{mH}=126 \mathrm{GeV}$ for the individual channels and their combination. [7].

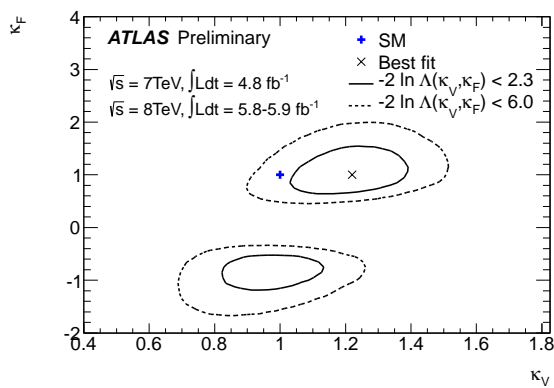

Figure 6. Fits for 2-parameter benchmark models probing different coupling strength scale factors for fermions and vector bosons; in this case the correlation of the coupling scale factors $\kappa_{F}$ and $\kappa_{V}$, assuming no non-SM contribution to the total width [7]. 